\title{
Antitumor effects of Tubeimoside-1 in NCI-H1299 cells are mediated by microRNA-126-5p-induced inactivation of VEGF-A/VEGFR-2/ERK signaling pathway
}

\author{
HANBING SHI $^{*}$, HONGXIA BI ${ }^{2 *}$, XINGYUAN SUN ${ }^{3}$, HAIYING DONG ${ }^{4}$, YUNFEI JIANG ${ }^{1}$, \\ HAIJUN MU ${ }^{1}$, GUOHUA LIU ${ }^{1}$, WEILI KONG ${ }^{1}$, RUIZHI GAO ${ }^{1}$ and JIANG SU ${ }^{5}$ \\ ${ }^{1}$ Department of Respiration II, The Third Affiliated Hospital of Qiqihar Medical University; ${ }^{2}$ Department of \\ Respiratory Medicine, The Hospital of Qiqihar Medical University; ${ }^{3}$ Department of Neurology, \\ The Third Affiliated Hospital of Qiqihar Medical University; ${ }^{4}$ Laboratory Center of Ultrastructural Pathology; \\ ${ }^{5}$ Department of Cardiovascular Medicine, Qiqihar Medical University, Qiqihar, Heilongiiang 161006, P.R. China
}

Received June 23, 2017; Accepted January 2, 2018

DOI: $10.3892 / \mathrm{mmr} .2018 .8459$

\begin{abstract}
Tubeimoside-1 (TBMS1), a triterpenoid saponin isolated from the tuber of Bolbostemma paniculatum (Maxim) Franquet, serves an universal suppressive role in multiple cancer types, including lung cancer. However, the mechanism involved in non-small cell lung cancer (NSCLC) cells by which TBMS1 elicits its antitumor effects is not yet completely understood. The present study indicated that $10 \mu \mathrm{mol} / \mathrm{l}$ TBMS1 significantly enhanced apoptosis and notably blocked the migration and invasion of NCI-H1299 cells. These effects were reversed following transfection with miR-126-5p inhibitor into TBMS1-treated NCI-H1299 cells. Vascular endothelial growth factor-A (VEGF-A) is a target gene for miR-126-5p. Notably, results suggested that the downregulated VEGF-A and VEGFR-2 in TBMS1-treated NCI-H1299 cells were upregulated after inhibiting miR-126-5p, and overexpression of VEGF-A or VEGFR-2 could significantly reduce apoptosis and promote the migration and invasion of TBMS1-treated NCI-H1299 cells. Furthermore, TBMS1 combined with TBHQ (an ERK activator) dramatically suppressed TBMS1-induced apoptosis and stimulated TBMS1-reduced migration and
\end{abstract}

Correspondence to: Dr Jiang Su, Department of Cardiovascular Medicine, Qiqihar Medical University, 333 North Bukui Street, Qiqihar, Heilongjiang 161006, P.R. China

E-mail: jiang_su2016@sina.com

${ }^{*}$ Contributed equally

Abbreviations: TBMS1, tubeimoside-1; VEGF-A, vascular endothelial growth factor-A; VEGF-2, vascular endothelial growth factor receptor-2; ERK, extracellular signal-regulated kinase; NSCLC, non-small cell lung cancer

Key words: tubeimoside-1, non-small cell lung cancer, microRNA-126-5p, vascular endothelial growth factor-A, extracellular signal-regulated kinase signaling pathway invasion in NCI-H1299 cells, suggesting that TBMS1 inhibits the ERK signaling pathway and represses the growth and metastasis of NCI-H1299 cells. Further study demonstrated that either inhibiting miR-126-5p or overexpressing VEGF-A and VEGFR-2 in TBMS1-treated NCI-H1299 cells elevated the mRNA expression levels and phosphorylation levels of MEK1, as well as ERK. To conclude, TBMS1 increases miR-126-5p expression, whereas overexpressing miR-126-5p inactivates VEGF-A/VEGFR-2/ERK signaling pathway, which ultimately actuates the pro-apoptotic and anti-metastatic effects in NCI-H1299 cells. Therefore, the present findings provide a theoretical foundation for TBMS1 as a potential candidate in NSCLC treatment.

\section{Introduction}

Lung cancer is the second most prevalent and the most lethal malignancy in both men and women throughout the world (1). The majority of which are non-small cell lung cancer (NSCLC) related to tobacco-driven carcinogenesis (2). NSCLC originates from respiratory epithelial cells (3), the early stage of which can be treated by curative intent surgery, but NSCLC is always un-diagnosable until advanced stage IIIB or IV because of its nonspecific early symptoms, or relapse after surgery because of its unlimited proliferation and metastasis (4). Generally, NSCLC cells could migrate to regional lymph nodes through lymphatic spreading and to distant organs through hematogenous spreading, most commonly to the liver, bone marrow, adrenal glands and the brain (1). Therefore, effective therapeutic approaches to control the proliferation and metastasis of NSCLC are expected to reduce mortality of this disease.

Tubeimoside-1 (TBMS1) is an active ingredient from the tuber of Bolbostemma paniculatum (Maxim) Franquet (5), the traditional Chinese herb with detoxification and detumescent properties (6). Prior studies have been identified that TBMS1 exerted potent antitumor activity with low toxicity to non-tumor cells (7). It can arrest the cell cycle at G2/M phase to inhibit proliferation $(6,8,9)$, as well as induce the release 
of cytochrome $c$ via increasing mitochondrial dysfunction and endoplasmic reticulum stress to enhance the apoptosis in various cancer cells (10-13). In lung cancer, TBMS1 exerts its cytotoxicity by increasing the Bax to $\mathrm{Bcl}-2$ ratio and triggering mitochondrial-related apoptotic pathway $(14,15)$. However, neither the contribution of TBMS1 to NSCLC metastasis nor the internal mechanism has been substantiated.

miRNAs are evolutionarily conserved non-coding RNAs capable of negatively regulating gene expression by binding to the 3'-untranslated region (3'-UTR) on target mRNAs (16). miR-126-5p, as known as miR-126*, is a $5^{\prime}$ part of the miR-126 transcript that located in the epidermal growth factor-like domain 7 (EGFL7) gene (17). miR-126 is involved in multiple processes of cellular activities via suppressing target genes such as vascular endothelial growth factor (VEGF), PI3K, EGFL7, CRK, ADAM9, IRS-1, SOX-2 and SLC7A5 (16). Previous studies have found that miR-126-5p was significantly downregulated in patients with coronary artery disease, overexpression of miR-126-5p promoted endothelial proliferation, increased pro-angiogenic endothelial cell behavior and limited atherosclerosis (18-20). Apart from that, the expression of miR-126-5p is notably reduced in alcohol-related hepatocellular carcinoma, breast carcinoma and prostate cancer $(21,22)$. Cho and colleagues showed that miR-126-5p was significantly downregulated using miRNA microarrays, which was the most differentially expressed miRNA in lung cancer (23). Accordingly, we speculated that TBMS1 might repress the progression of NSCLC through regulating the expression of miR-126-5p.

Therefore, this study observed the status of survival, migration and invasion in TBMS1-treated NCI-H1299 cells with miR-126-5p inhibitor transfection. Then we detected miR-126-5p targeted VEGF-A-related pathway through overexpression of VEGF-A and VEGFR-2 in TBMS1-treated NCI-H1299 cells. The results showed that the cytostatic and anti-metastatic effects of TBMS1 was mediated by overexpressing miR-126-5p caused inactivation of VEGF-A/VEGFR-2/ERK pathway.

\section{Materials and methods}

Cell culture. Human non small cell lung cancer NCI-H1299 cell line was purchased from Shanghai Institutes for Biological Sciences, Chinese Academy of Sciences (Shanghai, China). Cells were grown in Roswell Park Memorial Institute-1640 (RPMI-1640; Gibco, Grand Island, NY, USA) supplemented with $10 \%$ fetal bovine serum (FBS; HyClone, Logan, UT, USA) and streptomycin/penicillin $(100 \mathrm{U} / \mathrm{ml})$ at $37^{\circ} \mathrm{C}$ in a humidified atmosphere containing $5 \% \mathrm{CO}_{2}$.

Drug treatment. TBMS1 (Shanghai PureOne Biotechnology Co., Shanghai, China) was completely dissolved in $\mathrm{ddH}_{2} \mathrm{O}$. NCI-H1299 cells were exposed to TBMS1 of an ascending concentration range $(0,2.5,5,10,25,50 \mu \mathrm{M})$ for $48 \mathrm{~h}$ followed by CCK- 8 assay to find the optimum concentration. Then NCI-H1299 cells with or without transfection were exposed to $10 \mu \mathrm{mol} / 1 \mathrm{TBMS} 1$ for $48 \mathrm{~h}$ for further experiments. The untreated NCI-H1299 cells with or without transfection were experimented in parallel as control. For the detection of ERK pathway, NCI-H1299 cells were administrated with
$10 \mu \mathrm{mol} / 1 \mathrm{TBMS1}$ and tert-butylhydroquinone (TBHQ; Santa Cruz Biotechnology, Inc., Santa Cruz, CA, USA) for $48 \mathrm{~h}$. The TBMS1 treatment alone and untreated NCI-H1299 cells were employed as positive control and negative control.

Patients. We collected tumor tissues from 14 patients who underwent thoracoscopic lobectomy surgery for non-small cell lung cancer between May 2013 and January 2016 at the Third Affiliated Hospital of Qiqihar Medical University (Heilongjiang, China), and 14 paraneoplastic lung tissue samples ( $>5 \mathrm{~cm}$ away from tumors) were taken as healthy control. All tissue specimens were obtained with permission from the Medical Ethics Committee of The Third Affiliated Hospital of Qiqihar Medical University. The median age of all patients was 66.57 years (range, 43-78 years). None of the patients received chemotherapy, radiotherapy or immunotherapy before surgery. Each patient was agreed to participate in our study and signed an informed consent. All tissue specimens were obtained with permission from the Medical Ethics Committee of Qiqihar Medical University.

Cell Counting Kit-8 (CCK-8) assay. NCI-H1299 cells were seeded in 96 -well plates with a density of $1 \times 10^{4} /$ well and incubated to $80 \%$ confluence, followed by incubating with indicated concentrations of TBMS1 for $48 \mathrm{~h}$, with five replicates for each testing point including the negative control and blank wells. Thereafter, the cell growth was measured by Enhanced Cell Counting Kit-8 (Beyotime Institute of Biotechnology, Haimen, China) completely following the manufacturer's directions. Optical density (OD) values were evaluated at $450 \mathrm{~nm}$ by a microplate reader (BioTek Instruments, Inc., Winooski, VT, USA).

Plasmids, miR-126-5p inhibitor and transfection. The plasmids pcDNA3.1-VEGF-A and pcDNA3.1-VEGFR-2 harboring VEGF-A-coding sequences and VEGFR-2-coding sequences, along with miR-126-5p inhibitor were all constructed by Vipotion Co., Ltd. (Guangzhou, China) and were transfected into NCI-H1299 cells respectively using the Lipofectamine 2000 reagent (Invitrogen, Carlsbad, CA, USA) strictly following the manufacturer's instructions. Cells were harvested after $24 \mathrm{~h}$ of transfection for RT-qPCR detection and $48 \mathrm{~h}$ of transfection for western blot detection.

Hoechst staining assay. NCI-H1299 cells in each group were grown onto coverslips in 12 -well plates at a density of $5 \times 10^{4} /$ well. After growing to $80 \%$ confluences, cells were administrated with $10 \mu \mathrm{mol} / \mathrm{l}$ TBMS1 for $48 \mathrm{~h}$ followed by Hoechst Staining assay strictly according to the protocol of Hoechst Staining kit (Beyotime Institute of Biotechnology). Then all coverslips were mounted inversely onto slides with anti-fluorescein quencher addition (Beijing Solarbio Science \& Technology Co., Ltd., Beijing, China) and observed under a fluorescence microscope (Olympus, Tokyo, Japan) and photographed.

Flow cytometric analysis of cell apoptosis. Cell apoptosis analysis was performed with a Annexin V-FITC/PI apoptosis detection kit (Nanjing KeyGen Biotech Co., Ltd., Nanjing, China) strictly following the manufacturer's instructions. In 
brief, Cells in each group was resuspended in $500 \mu \mathrm{l}$ binding buffer containing $5 \mu \mathrm{l}$ Annexin V-FITC and $5 \mu \mathrm{l}$ PI. Then the mixture was incubated for $15 \mathrm{~min}$ at room temperature in the dark. Cell apoptosis was detected through flow cytometry and calculated by CellQuest software.

Wound healing assay. Cells were planted in 6-well plates and grown to $80 \%$ confluence. A artificial wound was gently created with a $200 \mu 1$ pipette tip on the confluent cell monolayer in each group. The detached cells were removed by FBS-free RPMI-1640 medium. Thereafter, cells were cultured in FBS free PMI-1640 medium supplemented with indicated drug for $48 \mathrm{~h}$. The migrating cells were imaged using an inverted microscope. The migration rate was the ratio of the migrated distance to the initial distance.

Matrigel-based invasion assay. The upper chambers of 24-well Transwell system (Corning, Tewksbury, MA, USA) were pre-coated with matrigel (BD Biosciences, San Jose, CA, USA). Cells were resuspended in FBS-free RPMI-1640 medium with indicated drug and plated in the upper chamber of the transwell system with a density of $2 \times 10^{4} /$ well. RPMI-1640 $(800 \mu \mathrm{l})$ plus $30 \% \mathrm{FBS}$ was added into the lower chamber. Cells were incubated in the Transwell system for $48 \mathrm{~h}$, then the non-invading cells at the upper-surface of the membrane were removed by cotton swabs, and the invading cells at the under-surface of the membrane were fixed in $4 \%$ paraformaldehyde for $20 \mathrm{~min}$ and stained with crystal violet for $5 \mathrm{~min}$. The number of invading cells at each group was counted in five randomly selected fields with a blinded manner under an inverted microscope.

Reverse transcription-quantitative polymerase chain reaction (RT-qPCR). Total RNA in each sample was extracted by an RNA extraction kit (Tiangen Biotech Co., Ltd., Beijing, China) completely following the manufacturer instructions and reverse-transcribed into cDNA. RT-qPCR was carried out by Stratagene Mx3000P (Stratagene; Agilent Technologies, Inc., Santa Clara, CA, USA) using Bestar ${ }^{\circledR}$ SybrGreen qPCR MasterMix (DBI ${ }^{\circledR}$ Bioscience, Ludwigshafen, Germany) with the following protocol: Initial denaturation at $95^{\circ} \mathrm{C}$ for $2 \mathrm{~min}$, 40 cycles consisting of $94^{\circ} \mathrm{C}$ for $20 \mathrm{sec}, 58^{\circ} \mathrm{C}$ for $20 \mathrm{sec}$, and $72^{\circ} \mathrm{C}$ for $20 \mathrm{sec}$. The following primers were used: miR-126-5p, 5'-CTCAACTGGTGTCGTGGAGTCGGCAATTCAGTTGAG CGCGTA-3' (sense) and 5'-ACACTCCAGCTGGGCATTA TTACTTTTGGTA-3' (antisense); VEGFR-A, 5'-AGGGCAG AATCATCACGAAGT-3' (sense) and 5'-AGGGTCTCGATT GGAT-GGCA-3' (antisense); VEGFR-2, 5'-ATAGAAGGTG CCCAGGAAAAG-3' (sense) and 5'-GTCTTCAGTTCCCCT CCATTG-3' (antisense); MEK1, 5'-GGGCTTCTA-TGGTGC GTTCTA-3' (sense) and 5'-CCCACGGGAGTTGACTAG GAT-3' (antisense); ERK, 5'-TCTGGAGCAGTATTACGA CCC-3' (sense) and 5'-CTGGCTGGAATCTAGCAGTCT-3' (antisense); $\beta$-actin, 5'-ATCGTGCGTGACA-TTAAGGAGA AG-3' (sense) and 5'-AGGAAGGAAGGCTGGAAGAGTG-3' (antisense). Relative mRNA expressions were obtained by $2^{-\Delta \Delta C T}$ method. $\beta$-actin was served as an internal control.

Western blot. Cells in each group were lysed with NP-40 lysate (Beyotime Institute of Biotechnology) including 1\% phenylmethanesulfonyl fluoride (PMSF). The concentration of total proteins was quantitated by a bicinchoninic acid (BCA) protein assay kit (Beyotime Institute of Biotechnology). Then equal amounts $(20 \mu \mathrm{g})$ of different proteins were loaded and separated using SDS-polyacrylamide gel electrophoresis (PAGE) followed by electrotransferred onto polyvinylidene fluoride (PVDF) membranes (Millipore, Bedford, MA, USA). After being blocked with $5 \%$ non-fat milk at $4{ }^{\circ} \mathrm{C}$ overnight, the membranes were probed with specific primary antibodies against VEGFR-2, p-VEGFR-2 (Tyr1175), ERK1/2, p-ERK1 (pT202/pY204) + p-ERK2 (pT185/pY187) (all 1:1,500 diluted); and VEGF-A, MEK1, p-MEK1 (pS298) (all 1:1,000 diluted; Abcam, Cambridge, MA, USA Abcam) at $4^{\circ} \mathrm{C}$ overnight. The membranes were then incubated with the secondary goat anti-rabbit IgG-HRP antibody (1:20,000 diluted; Wuhan Boster Biological Technology, Ltd., Wuhan, China) at $37^{\circ} \mathrm{C}$ for $40 \mathrm{~min}$. The unbound antibodies in each step were washed with TBST for three times. The target bands were visualized by an enhanced chemiluminescence (ECL; Millipore), and the protein intensities were detected by Gel-Pro Analyzer software (Media Cybernetics, Inc., Bethesda, MD, USA). GAPDH was used as an internal control.

Statistical analysis. Statistical analysis was performed with GraphPad Prism 5.0 software. All data are presented as mean \pm standard deviation (SD). Differences between groups in RT-PCR detection of clinical tissues were calculated with unpaired Student's t-test. Other differences comparison between groups were analyzed with one-way analysis of variance (ANOVA). $\mathrm{P}<0.05$ were considered to indicate a statistically significant difference.

\section{Results}

$10 \mu M$ TBMS1 could significantly reduce cell growth and upregulate miR-126-5p expression. We first employed CCK-8 assay to determine the effect of TBMS1 on NCI-H1299 cell growth. The results showed that cell viability exhibited dose-dependent inhibitions after TBMS1 treatment for $48 \mathrm{~h}$, and the inhibitory effect was sharply increased at $10 \mu \mathrm{M}$ TBMS1 treatment, so we chose $10 \mu \mathrm{M}$ TBMS1 for subsequent experiments (Fig. 1A; $\mathrm{P}<0.001$ ).

Next, we conducted RT-qPCR to detect the expression of miR-126-5p in both NSCLC pathological specimens and TBMS1-treated NCI-H1299 cells. We found that miR-126-5p was notably downregulated in NSCLC tissues compared with normal adjacent tissues (Fig. 1B; $\mathrm{P}<0.001$ ). Furthermore, the significantly elevated miR-126-5p level was observed in NCI-H1299 cells upon TBMS1 treatment for $48 \mathrm{~h}$ compared with control (Fig. 1C; $\mathrm{P}<0.001$ ).

The above results demonstrated that $10 \mu \mathrm{M}$ TBMS1 could significantly reduce cell growth and upregulate miR-126-5p expression.

TBMS1 stimulates the apoptosis and inhibits the metastasis of NCI-H1299 cells by promoting miR-126-5p expression. To identify whether miR-126-5p is involved in the antitumor effects of TBMS1 in NSCLC cells, the miR-126-5p inhibitor was transfected into NCI-H1299 cells. Then Hoechst staining and flow cytometry were employed to investigate 

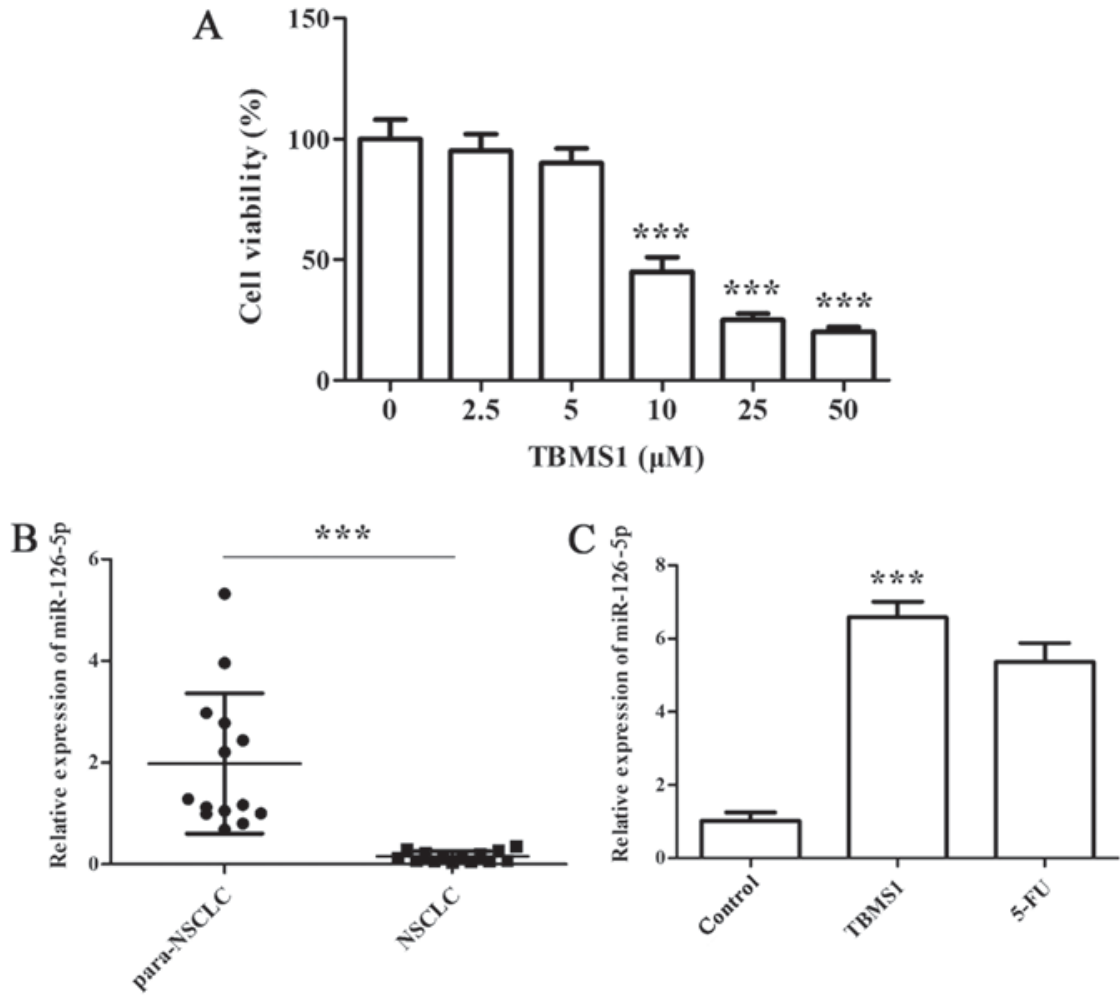

Figure 1. TBMS1 $(10 \mu \mathrm{M})$ could significantly reduce cell growth and upregulate miR-126-5p expression. (A) NCI-H1299 cells were seeded in 96-well plates and exposed to increased concentrations of TBMS1 $(0,2.5,5,10,25,50 \mu \mathrm{M})$ for $48 \mathrm{~h}$, followed by CCK- 8 assay. ${ }^{* * * *} \mathrm{P}<0.001$ vs. non-TBMS1 treated control. (B) RT-qPCR analysis of miR-126-5p in NSCLC tissues and paraneoplastic tissues. compared with para-NSCLC, ${ }^{* * *} \mathrm{P}<0.001$. (C) RT-qPCR analysis of miR-126-5p in NCI-H1299 cells with $10 \mu \mathrm{M}$ TBMS1 treatment for $48 \mathrm{~h}$. Non-TBMS1 treated control was used as negative control, and 5-FU treated cells was served as positive control. $\beta$-actin was used as an internal control. Above experiments were performed for three times. Data are expressed as mean \pm SD ${ }^{* * * *} \mathrm{P}<0.001$ vs. control. TBMS1, tubeimoside- 1 .

cell apoptosis, and wound healing and matrigel-based invasion assays were used to evaluate the migration and invasion of NCI-H1299 cells. The results showed that substantial nuclear condensation and apoptotic bodies were appeared in TBMS1-treated NCI-H1299 cells, which was reversed after inhibiting miR-126-5p (Fig. 2A; P<0.001). Similarly, the total apoptosis rate of NCI-H1299 cells was increased significantly after TBMS1 treatment for $48 \mathrm{~h}$ compared with control, but was decline after inhibiting miR-126-5p (Fig. 2B; $\mathrm{P}<0.001$ ). Besides, the wound healing rate of TBMS1 treated NCI-H1299 cells was much lower than control at the point of $48 \mathrm{~h}$ after wound creation $(\mathrm{P}<0.001)$, but was notably elevated after inhibiting miR-126-5p (Fig. 2C; P<0.05). Simultaneously, the number of invading cells was $19.10 \pm 2.40$ after TBMS1 treatment for $48 \mathrm{~h}$, which was obviously decreased as compared to control cells (58.16 \pm 5.03$)$, but the effect of TBMS1 was diminished by inhibiting miR-126-5p (Fig. 2D; P<0.001). The above results suggested that TBMS1 stimulates the apoptosis and inhibits the metastasis of NCI-H1299 cells by promoting miR-126-5p expression.

TBMS1 increased miR-126-5p downregulates the expressions of VEGF-A and VEGFR-2 to enhance the apoptosis and reduce the metastasis of NCI-H1299 cells. RT-qPCR and western blot analysis were performed to detect the expression of VEGF-A and VEGFR-2 in miR-126-5p inhibitor transfected NCI-H1299 cells under TBMS1 treatment. We found that TBMS1 coordinately downregulated VEGF-A and VEGFR-2 expressions at both mRNA and protein levels in NCI-H1299 cells compared with control, as well as the significantly suppressed phosphorylation of VEGFR-2 (Fig. 3A-C; $\mathrm{P}<0.001$ ). While inhibiting miR-126-5p remarkably reversed above effects (Fig. 3A-C; P<0.001). As VEGF-A is a target for miR-126-5p, the results implied a link between VEGF-A/VEGFR-2 axis and TBMS1 induced pro-apoptotic and anti-metastatic effects.

We then overexpressed VEGF-A and VEGFR-2 respectively in NCI-H1299 cells and conducted flow cytometry, wound healing and matrigel-based invasion assays to explore the apoptosis, migration and invasion of NCI-H1299 cells. NCI-H1299 cells transfected with pcDNA3.1-VEGF-A and pcDNA3.1-VEGFR-2 displayed 3.80-fold and 2.94-fold higher mRNA levels along with 4.03-fold and 2.54-fold higher protein levels than those with respective pcDNA3.1 vectors transfected (Fig. 3D-F; P<0.001), indicating successfully overexpressed VEGF-A and VEGFR-2 in NCI-H1299 cells. Further study revealed that the apoptosis rates were decreased significantly in VEGF-A-overexpressing and VEGFR-2-overexpressing NCI-H1299 cells respectively than in non-transfected cells following the same TBMS1 treatment (Fig. 3G; P<0.01). As expected, the migration rates were notably elevated to 1.4 - and 1.5-fold after $48 \mathrm{~h}$ of TBMS1 treatment in VEGF-A-overexpressing and VEGFR-2-overexpressing NCI-H1299 cells respectively compared with non-transfected TBMS1-treated cells (Fig. 3H; P<0.05). Meanwhile, overexpressing VEGF-A and VEGFR-2 stimulated the invasion of 

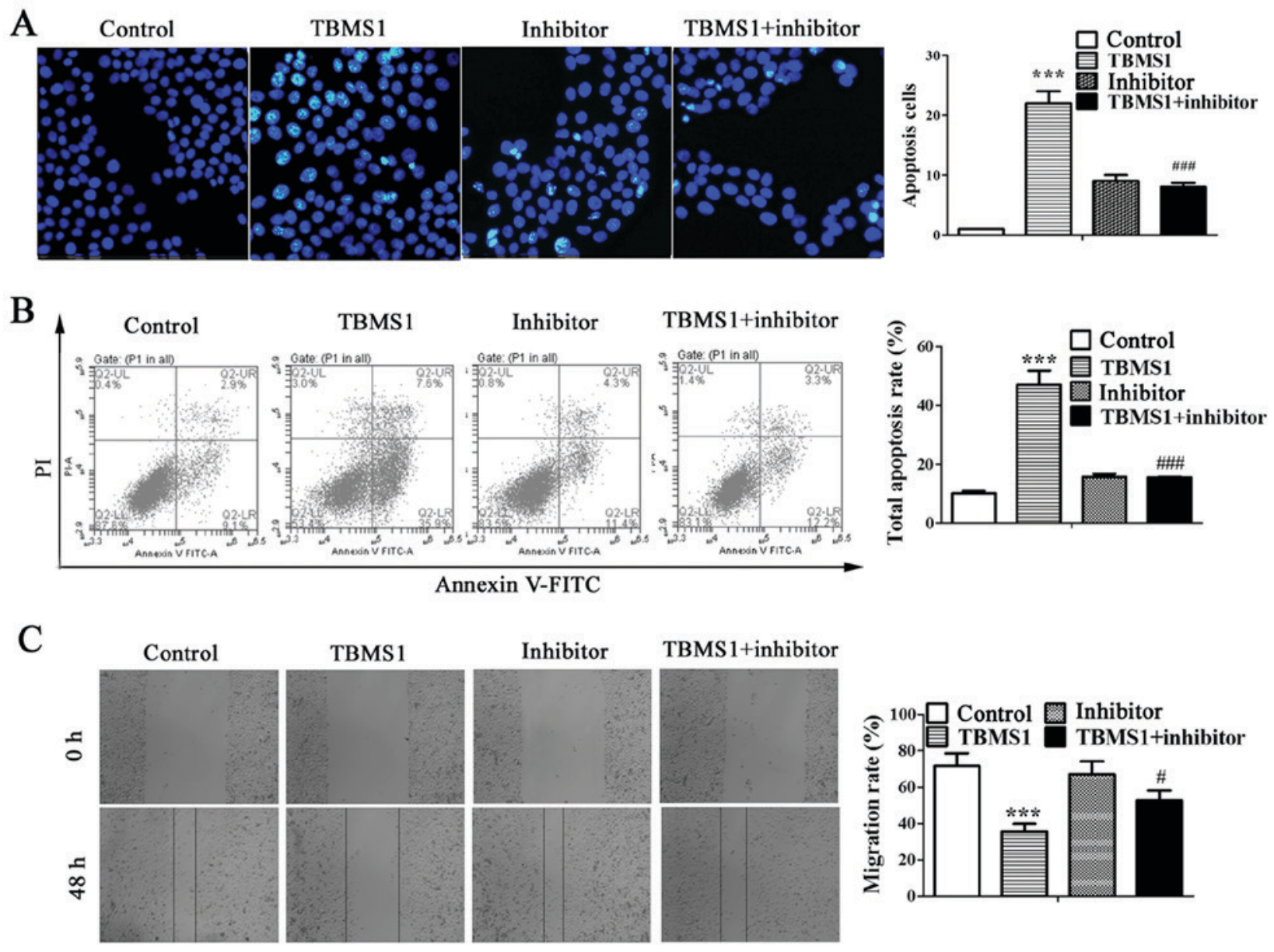

TBMS1+inhibitor

$\mathrm{D}$
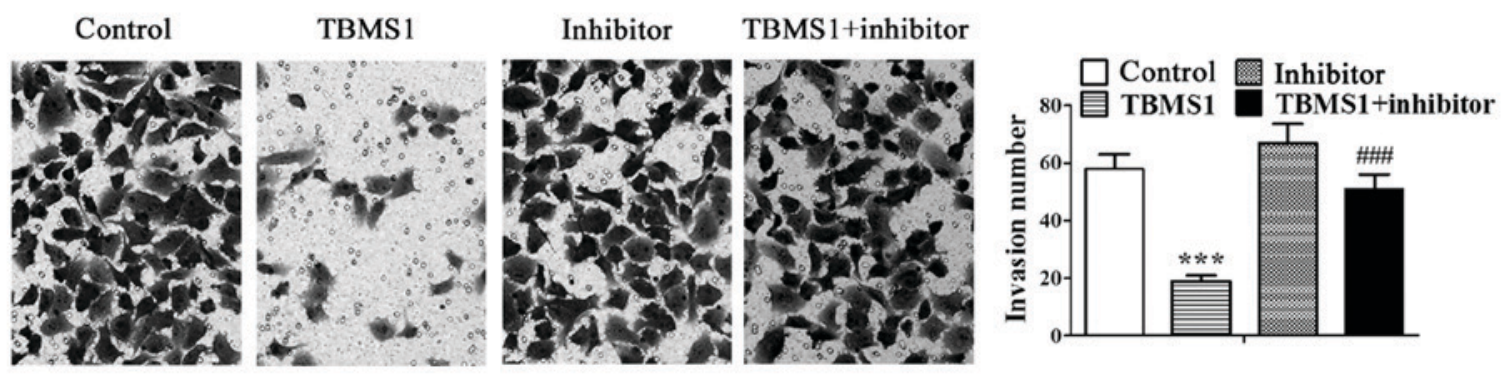

Figure 2. TBMS1 stimulates the apoptosis and inhibits the metastasis of NCI-H1299 cells by promoting miR-126-5p expression. NCI-H1299 cells were transfected with or without miR-126-5p inhibitor before incubating with $10 \mu \mathrm{mol} / 1 \mathrm{TBMS} 1$ for $48 \mathrm{~h}$. (A) Hoechst staining assay was performed to observe cell apoptosis at x200 magnification. Healthy cells were stained to be gray, and apoptotic cells were stained to be bright white. The apoptosis cells were counted as a bar chart. (B) Cell apoptosis was carried out using Annexin V-FITC/propidium iodide co-stained flow cytometry. The total apoptosis rate is summarized as a bar chart. (C) Cellular migration was evaluated by wound healing assay under an inverted microscope with $\times 200$ magnification after $48 \mathrm{~h}$ of incubation with TBMS1. (D) Cellular invasion was assessed through a Matrigel-coated Transwell system and observed under an inverted microscope with x 200 magnification after $48 \mathrm{~h}$ of incubation with TBMS1. The above four experiments were repeated three times. Representative photographs are shown. Data are represented as mean $\pm \mathrm{SD},{ }^{* * *} \mathrm{P}<0.001$ vs. control; ${ }^{\#} \mathrm{P}<0.05,{ }^{\# \#} \mathrm{P}<0.001$ vs. TBMS1. TBMS1, tubeimoside-1.

TBMS1-treated NCI-H1299 cells (Fig. 3I; P<0.01). Thus, the above results indicated that TBMS1-driven miR-126-5p enhanced the apoptosis and reduced the metastasis of NCI-H1299 cells through downregulating VEGF-A/VEGFR-2 axis.

TBMS1 inactivates ERK pathway to enhance the apoptosis and reduce the metastasis of NCI-H1299 cells. TBHQ is a phenolic antioxidant functioning as an activator of ERK, and it requires the help from upstream signaling kinase MAPK/ERK kinase (MEK). To deduce changes in ERK1/2 pathway as a function of TBMS1, NCI-H1299 cells were exposed to $10 \mu \mathrm{mol} / 1 \mathrm{TBMS} 1$ for $48 \mathrm{~h}$ combined with TBHQ, then flow cytometry, wound healing and matrigel-based invasion assays were used to detect the apoptosis, migration and invasion of NCI-H1299 cells. The results showed that the elevated apoptosis rate of NCI-H1299 cells with TBMS1 treatment was sharply declined after combination with TBHQ (Fig. 4A; $\mathrm{P}<0.001)$. In addition, the migration rate and invasion number in TBMS1-treated NCI-H1299 cells upon addition of TBHQ were increased significantly compared with TBMS1 treatment alone (Fig. 4B and C; $\mathrm{P}<0.01$ ). These results indicated that 

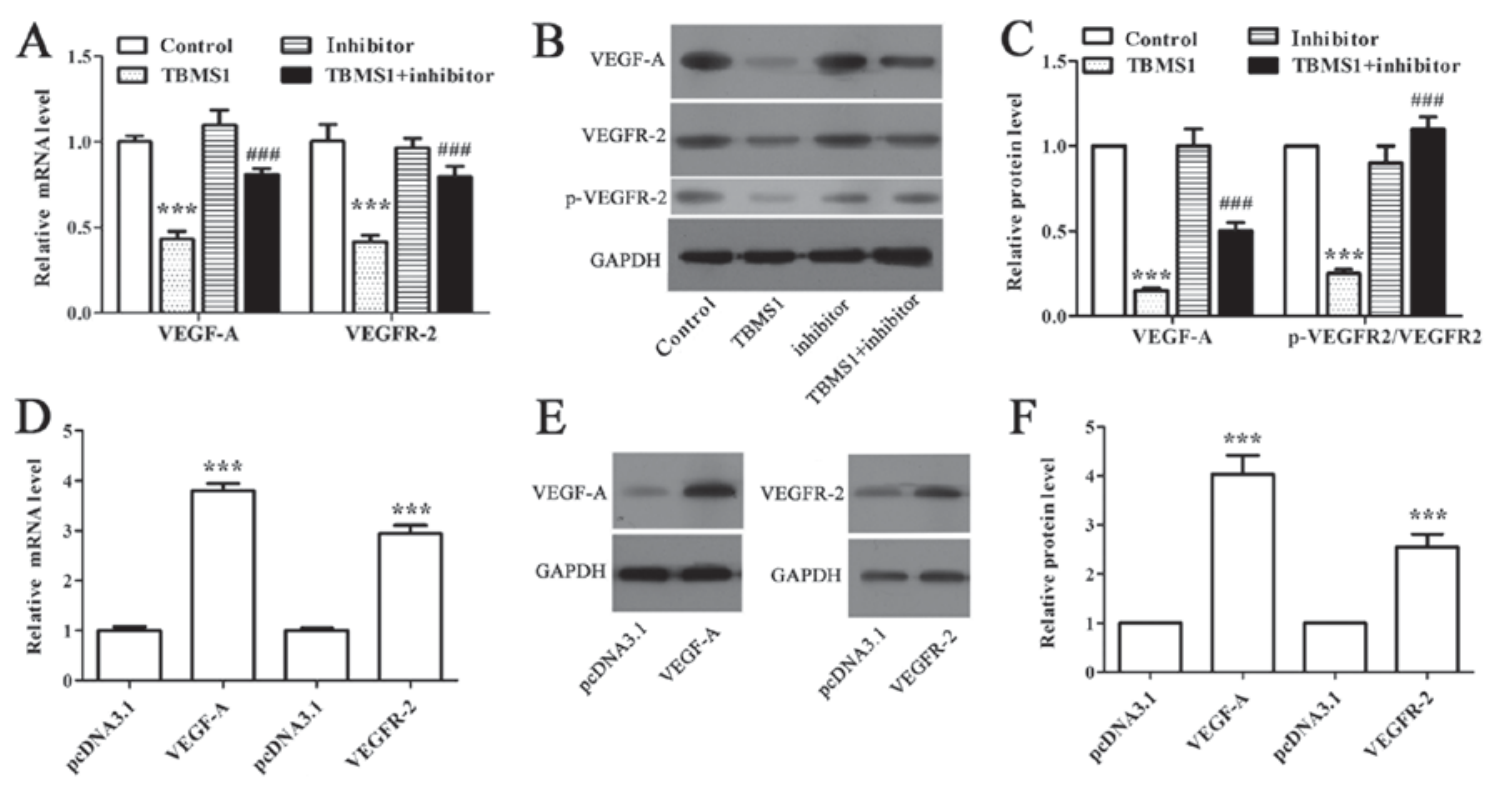

$\mathrm{E}$

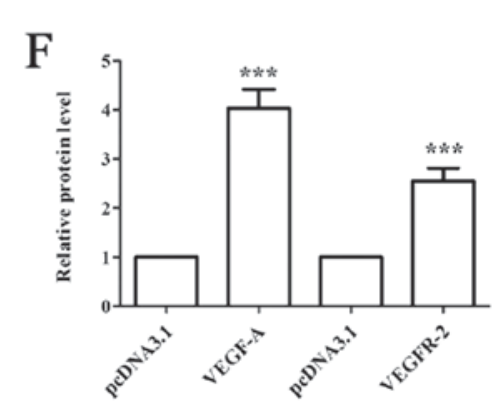

G
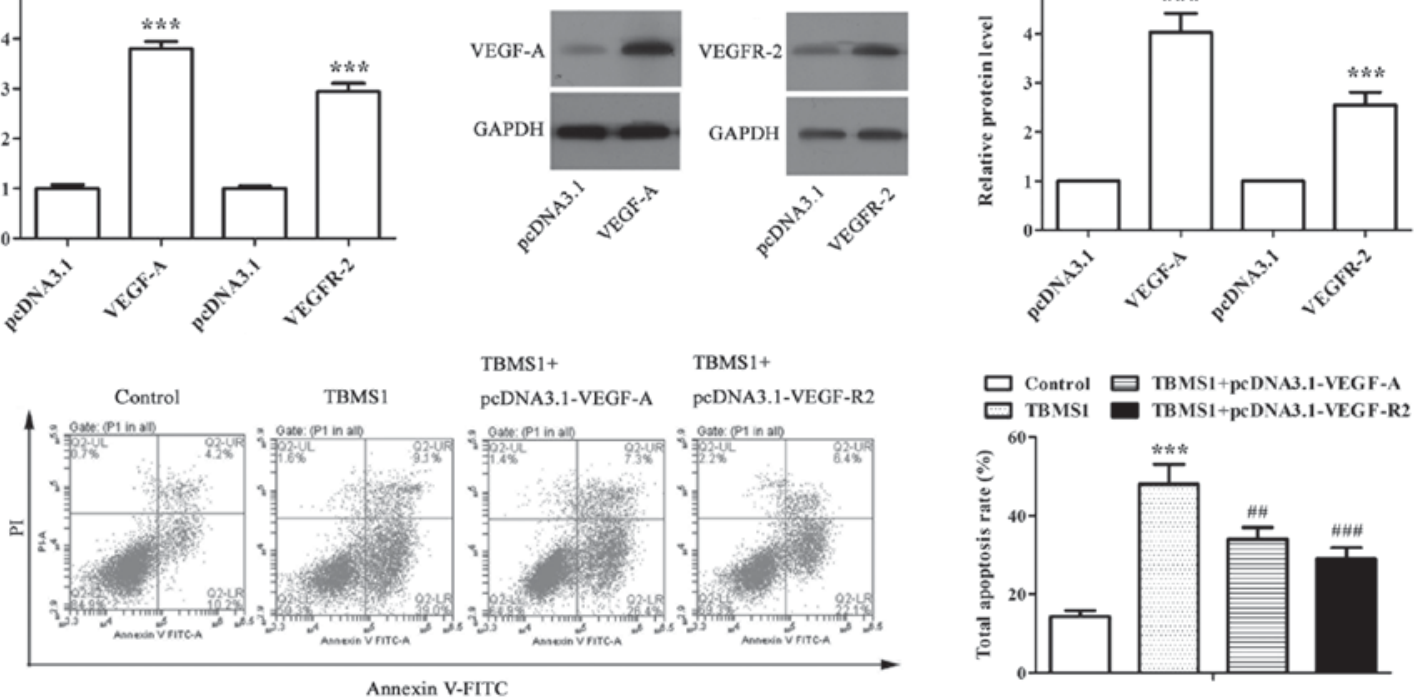

$\mathrm{H}$
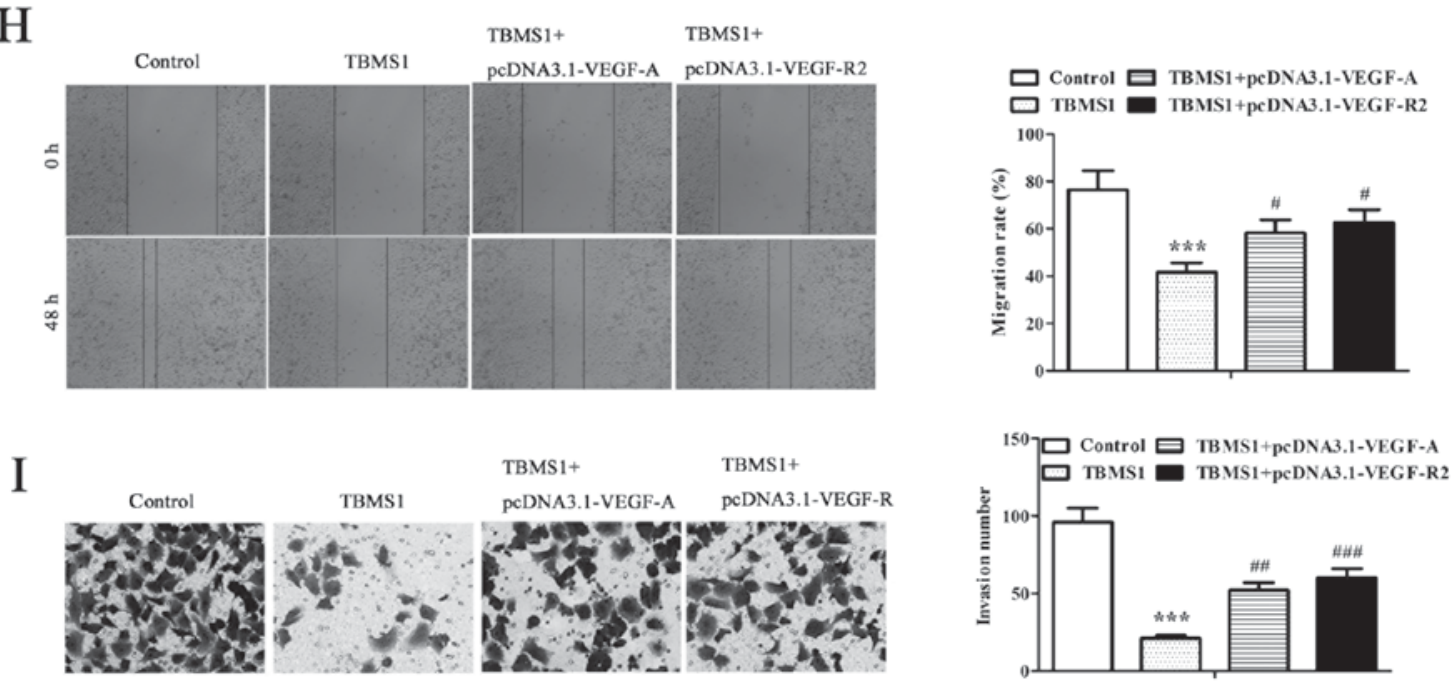

Figure 3. TBMS1 increased miR-126-5p downregulates the expressions of VEGF-A and VEGFR-2 to enhance the apoptosis and reduce the metastasis of NCI-H1299 cells. (A) RT-qPCR analysis or (B and C) western blot analysis on VEGF-A and VEGFR-2 mRNA, protein or phosphorylation (Tyr1175) levels in TBMS1-treated NCI-H1299 cells with or without miR-126-5p inhibitor transfection. $\beta$-actin for RT-qPCR, GADPH for western blot analysis were used as internal controls. ${ }^{* * *} \mathrm{P}<0.001$ vs. control; ${ }^{\# P}<0.05$, ${ }^{\# \# \#} \mathrm{P}<0.001$ vs. TBMS1. (D) RT-qPCR analysis of VEGF-A and VEGFR-2 mRNA expression levels. $\beta$-actin was used as an internal control. ${ }^{* * *} \mathrm{P}<0.001$ vs. corresponding pcDNA3.1. (E) Representative images of western blot analysis on VEGF-A and VEGFR-2 protein expression levels. GADPH was used as an internal control. (F) Intensity comparison of western blot analysis. ${ }^{* * *} \mathrm{P}<0.001$ vs. corresponding pcDNA3.1. (G) Annexin V-FITC/PI stained flow cytometry analysis; (H) wound healing experiment (magnification, x40); and (I) Tranwell invasion assay (magnification, x200) were carried out in NCI-H1299 cells after $10 \mu \mathrm{mol} / 1$ TBMS1 treatment for $48 \mathrm{~h}$. A series of representative images of corresponding results are shown. ${ }^{* * *} \mathrm{P}<0.001$ vs. control; ${ }^{~} \mathrm{P}<0.05,{ }^{\# \#} \mathrm{P}<0.01,{ }^{\# \# \#} \mathrm{P}<0.001$ vs. TBMS1. All data in each group are expressed as mean \pm SD from three independent experiments. TBMS1, tubeimoside-1; VEGF-A, vascular endothelial growth factor-A.

TBMS1 could inactivate ERK pathway to enhance the apoptosis and reduce the metastasis of NCI-H1299 cells.

The pro-apoptotic and anti-metastatic effects of TBMS1 in NCI-H1299 cells are mediated by overexpressing miR-126-5p induced inactivation of VEGF-A/VEGFR-2/ERK pathway. We employed RT-qPCR and Western blot to address whether ERK1/2 pathway is the downstream mediator of miR-126-5p downregulated VEGF-A/VEGFR-2. We discovered that both mRNA and phosphorylation levels of MEK1 and ERK were 

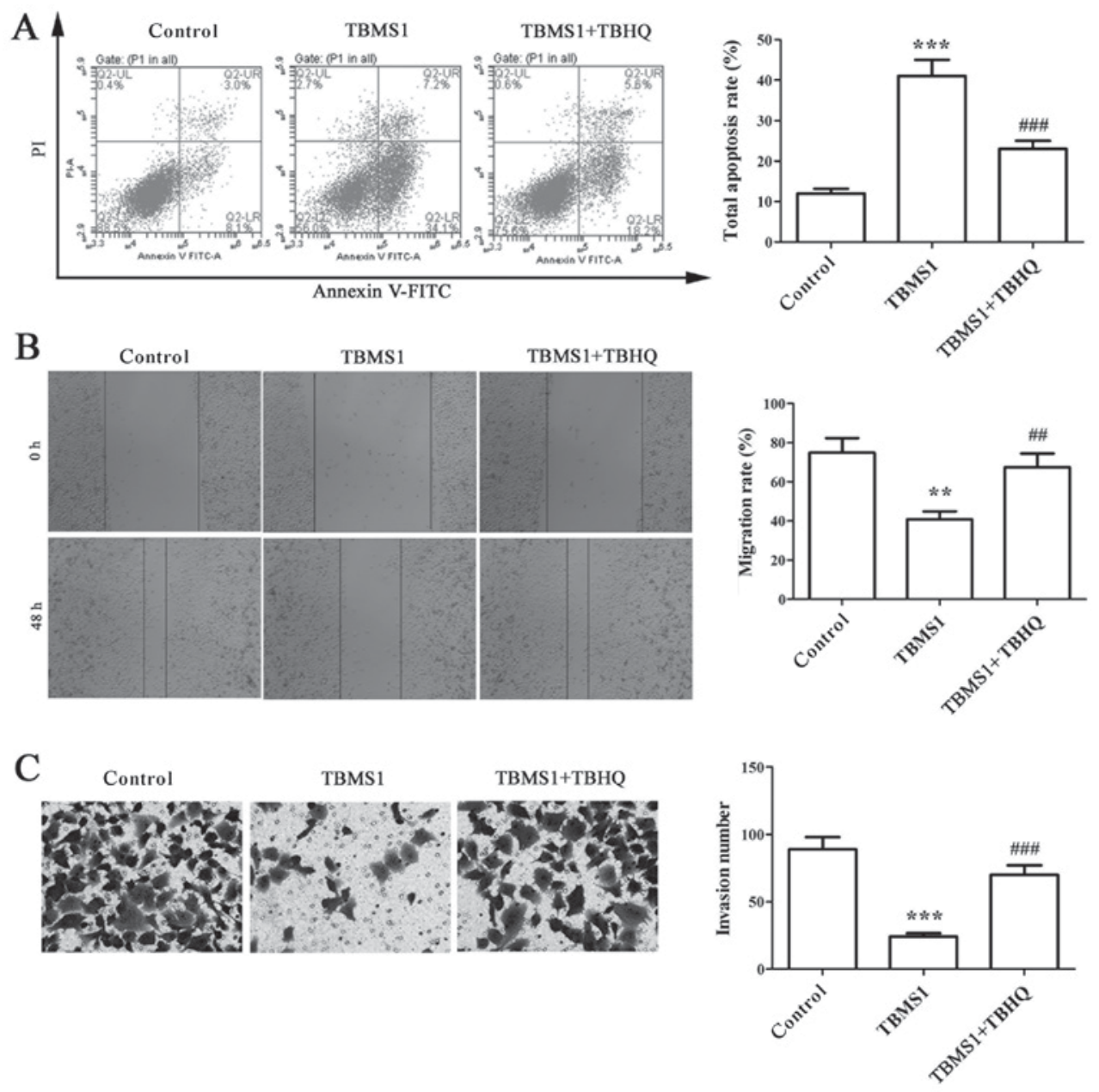

Figure 4. TBMS1 inactivates ERK pathway to enhance the apoptosis and reduce the metastasis of NCI-H1299 cells. NCI-H1299 cells co-cultured with TBMS1 and TBHQ or incubated with TBMS1 alone for $48 \mathrm{~h}$ were subjected to (A) flow cytometry analysis of cell apoptosis. (B) Wound healing analysis of cell migration (magnification, $\mathrm{x} 40$ ). (C) Tranwell invasion assay (magnification, $\mathrm{x} 200$ ). The above results are given as mean \pm SD, the error bars represent the $\mathrm{SD}$ of three independent experiments, and representative examples of images are shown. ${ }^{* *} \mathrm{P}<0.01,{ }^{* * * *} \mathrm{P}<0.001$ vs. control; ${ }^{\# \#} \mathrm{P}<0.01,{ }^{\# \# "} \mathrm{P}<0.001 \mathrm{vs}$. TBMS1. TBMS1, tubeimoside-1.

declined significantly in NCI-H1299 cells upon TBMS1 treatment for $48 \mathrm{~h}$ in comparison with control (Fig. 5A-C; $\mathrm{P}<0.001$ ), while inhibiting miR-126-5p prevented this decline (Fig. 5A-C; $\mathrm{P}<0.05$ ). Next, we detected that overexpressing VEGF-A or VEGFR-2 in TBMS1-treated NCI-H1299 cells caused the elevated mRNA and phosphorylation levels of both MEK1 and ERK (Fig. 5D-F; P<0.05). Taken together, we concluded that the pro-apoptotic and anti-metastatic effects of TBMS1 in NCI-H1299 cells are mediated by overexpressing miR-126-5p induced inactivation of VEGF-A/VEGFR-2/ERK axis.

\section{Discussion}

Our study demonstrate that TBMS1 boosts the apoptosis and inhibits the migration and invasion of NSCLC cells by overexpressing miR-126-5p induced inactivation of VEGF-A/VEGFR-2/ERK pathway, highlighting a therapeutic pathway of TBMS1.

Clinical researches proved that the reduced miR-126-5p is the most differentially expressed miRNA in lung cancers (24). In addition, Felli et al revealed that overexpression of miR-126-5p actuated the reduction of proliferation, invasion, propagation and chemotaxis in melanoma cells (25). In the present study, we detected the significantly downregulated miR-126-5p in NSCLC tumor tissues, but the expression of miR-126-5p was raised much higher after TBMS1 administration in NCI-H1299 cells, we therefore speculate that TBMS1 may upregulate miR-126-5p to induce antitumor effects in NSCLC cells. Then we found that the pro-apoptotic and anti-metastatic effects of TBMS1 were notably abrogated by inhibition of miR-126-5p in NCI-H1299 cells, which results proved our conjecture.

Except for angiogenesis, VEGF-A-stimulated response also contains the invasion, migration, and survival of cancer (26-28), which is largely attributed to VEGFR-2 for its potent tyrosine kinase activity (29). VEGF-A binding to VEGFR-2 induces dimerization and transautophosphorylation of cytoplasmic tyrosine residues to trigger diverse cellular responses $(29,30)$. Overexpression of VEGF-A has been implicated in most human tumors, including NSCLC, and is associated with increased tumor recurrence, metastasis, and poorer prognosis (31-36). It is a well-established fact that VEGF-A is a direct miR-126-5p target, and miR-126-downregulated VEGF could inhibit the proliferation of lung cancer cells (37). Accordingly, we found that TBMS1 downregulated 
A

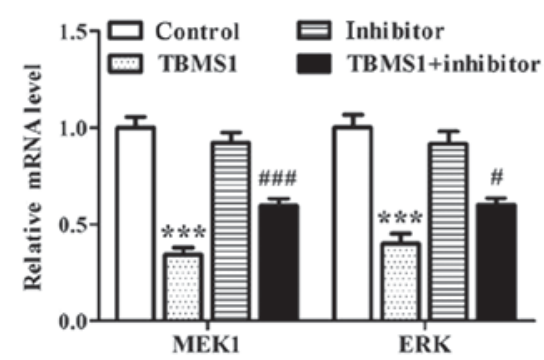

D

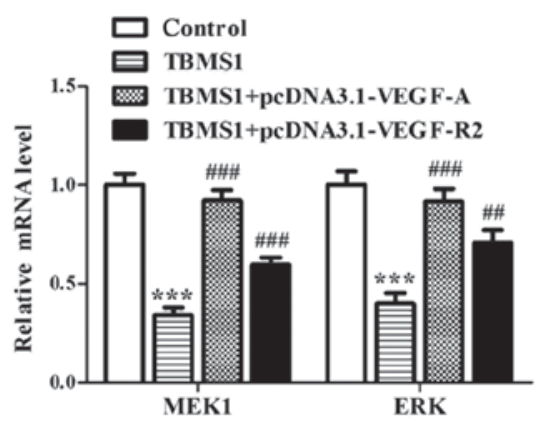

B

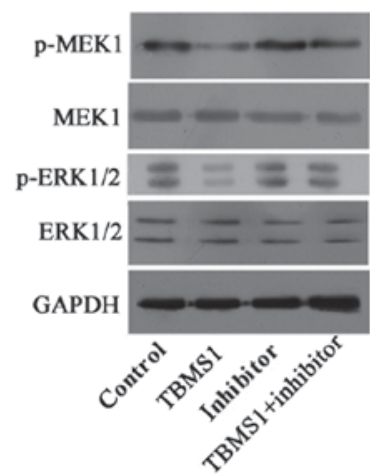

E

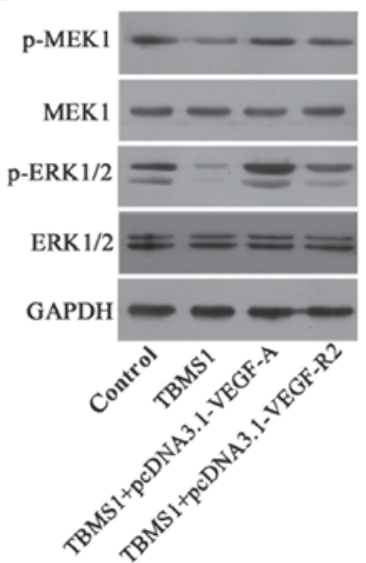

C

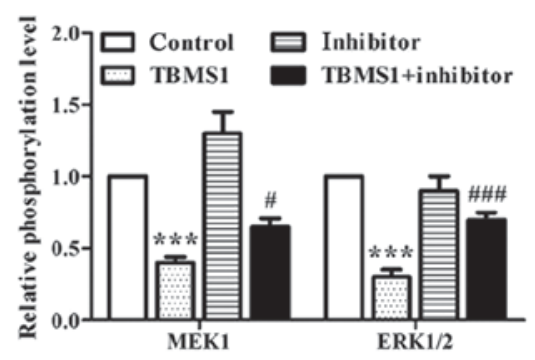

F

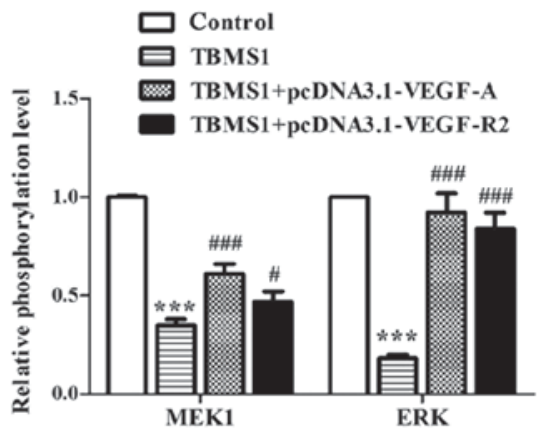

Figure 5. The pro-apoptotic and anti-metastatic effects of TBMS1 in NCI-H1299 cells are mediated by overexpressing miR-126-5p induced inactivation of VEGF-A/VEGFR-2/ERK pathway. NCI-H1299 cells were transfected with or without miR-126-5p inhibitor before TBMS1 treatment for 48 h, followed by RT-qPCR analysis of MEK1 and ERK mRNA expression levels (A), as well as western blot analysis of (B and C) MEK1 and ERK phosphorylation levels. Representative blots are shown. Overexpressing VEGF-A or VEGFR-2 NCI-H1299 cells were exposed to $10 \mu$ mol/1 TBMS1 treatment for 48 h, followed by RT-qPCR analysis of MEK1 and ERK mRNA expression levels (D), as well as western blot analysis of (E and F) MEK1 and ERK phosphorylation levels. Representative blots are shown. Experiments were done in triplicates for statistical significance, and all results are expressed as mean \pm SD. ${ }^{* * *} \mathrm{P}<0.001$ vs. control; ${ }^{\# \#} \mathrm{P}<0.05,{ }^{\# \#} \mathrm{P}<0.01,{ }^{\# \# \#} \mathrm{P}<0.001$ vs. TBMS1. TBMS1, tubeimoside- 1 .

VEGF-A through miR-126-5p, and overexpressing VEGF-A prevented TBMS1-mediated, miR-126-5p-dependent antitumor effects in NCI-H1299 cells, overexpressing VEGFR-2 yielded similar results, indicating that VEGF-A/VEGFR-2 are the downstream pathway of TBMS1 induced overexpression of miR-126-5p.

ERK1/2 pathway is an important member of MAPKs pathway capable of promoting growth, invasion, angiogenesis and metastasis by increasing the secretion of MMP-9 and the phosphorylation of ATF-2 (38-40). Liu et al identified that TBMS1 sensitizes cisplatin in cisplatin-resistant human ovarian cancer cells through downregulation of ERK and upregulation of p38 signaling pathways (41). In NSCLC, we discovered that inhibiting ERK pathway in TBMS1 treated NCI-H1299 cells could resulted in the pro-apoptotic and anti-metastatic effects, but above inactivation of ERK pathway was prevented upon inhibiting miR-126-5p, confirming that ERK pathway is also the downstream pathway of TBMS1-mediated, miR-126-5p-dependent antitumor effects.

VEGF-A/VEGFR-2/MEK1/ERK1/2 signaling pathway converge on the nucleus leading to DNA replication, cellular proliferation and migration. In vitro study demonstrated that overexpression of VEGF-A augmented cell proliferation and migration through this pathway, thereby inducing a more invasive tumor phenotype $(42,43)$. The phosphorylation of VEGFR2 at Tyr1175 induces PIP2 hydrolysis by activating PLC $\gamma$ to trigger ERK pathway. Silencing VEGFR-2 blocked the phosphorylation of ERK1/2 in lung cancer cells (44). Santos et al found that the intracellular VEGFR-2 inhibitor promoted apoptosis of acute myeloid leukemia cell lines through inhibiting ERK1/2 pathway (45). We found the reduced phosphorylation level of VEGFR2 under TBMS1 treatment, and further discovered that the inactivation of ERK pathway induced by TBMS1 was prevented when VEGF-A or VEGFR-2 was overexpressed in NCI-H1299 cells, indicating that VEGF-A/VEGFR-2/MEK1/ERK1/2 axis act as a direct mediator of TBMS1-mediated, miR-126-5p-dependent antitumor effects.

In conclusion, our present study gradually recognized and confirmed that TBMS1 could increase the expression of miR-126-5p, then miR-126-5p targeted VEGF-A inactivated the VEGF-A/VEGFR-2/ERK1/2 pathway to boost apoptosis and suppress migration and invasion in NCI-H1299 cells. Our data explained the mechanism of TBMS1-mediated antitumor effects in NCI-H1299 cells. TBMS1 may become a promising candidate for NSCLC therapy. 


\section{References}

1. Wald O, Shapira OM and Izhar U: CXCR4/CXCL12 axis in non small cell lung cancer (NSCLC) pathologic roles and therapeutic potential. Theranostics 3: 26-33, 2013

2. Kumarakulasinghe NB, van Zanwijk N and Soo RA: Molecular targeted therapy in the treatment of advanced stage non-small cell lung cancer (NSCLC). Respirology 20: 370-378, 2015.

3. Travis WD, Brambilla E, Noguchi M, Nicholson AG, Geisinger KR, Yatabe Y, Beer DG, Powell CA, Riely GJ, Van Schil PE, et al: International association for the study of lung cancer/american thoracic society/european respiratory society international multidisciplinary classification of lung adenocarcinoma. J Thorac Oncol 6: 244-285, 2011

4. Fennell DA, Summers Y, Cadranel J, Benepal T, Christoph DC, Lal R, Das M, Maxwell F, Visseren-Grul C and Ferry D: Cisplatin in the modern era: The backbone of first-line chemotherapy for non-small cell lung cancer. Cancer Treat Rev 44: 42-50, 2016.

5. Gu Y, Körbel C, Scheuer C, Nenicu A, Menger MD and Laschke MW: Tubeimoside-1 suppresses tumor angiogenesis by stimulation of proteasomal VEGFR2 and Tie 2 degradation in a non-small cell lung cancer xenograft model. Oncotarget 7 : 5258-5272, 2016

6. Yin Y, Chen W, Tang C, Ding H, Jang J, Weng M, Cai Y and Zou G: NF- $\mathrm{B}$, JNK and p53 pathways are involved in tubeimoside-1-induced apoptosis in HepG2 cells with oxidative stress and $\mathrm{G}_{2} / \mathrm{M}$ cell cycle arrest. Food Chem Toxicol 49: 3046-3054, 2011.

7. Yu L, Ma R, Wang Y and Nishino H: Potent anti-tumor activity and low toxicity of tubeimoside 1 isolated from Bolbostemma paniculatum. Planta Med 60: 204-208, 1994

8. Bartoli Klugman F, Decorti G, Candussio L, Mallardi F, Grill V, Zweyer $\mathrm{M}$ and Baldini L: Effect of ketotifen on adriamycin toxicity: Role of histamine. Cancer Lett 39: 145-152, 1988.

9. Xu Y, Wang G, Chen Q, Lin T, Zeng Z, Luo Q, Liu J and Sun C: Intrinsic apoptotic pathway and G2/M cell cycle arrest involved in tubeimoside I-induced EC109 cell death. Chin J Cancer Res 25: 312-321, 2013.

10. Jia G, Wang Q, Wang R, Deng D, Xue L, Shao N, Zhang Y, Xia X, Zhi F and Yang Y: Tubeimoside-1 induces glioma apoptosis through regulation of $\mathrm{Bax} / \mathrm{Bcl}-2$ and the ROS/Cytochrome C/Caspase-3 pathway. Onco Targets Ther 8: 303-311, 2015.

11. Xu Y, Chiu JF, He QY and Chen F: Tubeimoside-1 exerts cytotoxicity in HeLa cells through mitochondrial dysfunction and endoplasmic reticulum stress pathways. J Proteome Res 8: 1585-1593, 2009

12. Huang $\mathrm{P}, \mathrm{Yu} \mathrm{C}$, Liu XQ, Ding YB, Wang YX and He JL: Cytotoxicity of tubeimoside I in human choriocarcinoma JEG-3 cells by induction of cytochrome c release and apoptosis via the mitochondrial-related signaling pathway. Int J Mol Med 28 : 579-587, 2011

13. Wang F, Ma RD and Yu LJ: Role of mitochondria in tubeimoside I-mediated apoptosis in human cervical carcinoma HeLa cell line. Zhongguo Zhong Yao Za Zhi 30: 1935-1939, 2005 (In Chinese)

14. Lin Y, Xie G, Xia J, Su D, Liu J, Jiang F and Xu Y: TBMS1 exerts its cytotoxicity in NCI-H460 lung cancer cells through nucleolar stress-induced p53/MDM2-dependent mechanism, a quantitative proteomics study. Biochim Biophys Acta 1864: 204-210, 2016.

15. Zhang Y, Xu X and He P: Tubeimoside- 1 inhibits proliferation and induces apoptosis by increasing the Bax to Bcl-2 ratio and decreasing COX-2 expression in lung cancer A549 cells. Mol Med Rep 4: 25-29, 2011.

16. Ebrahimi F, Gopalan V, Smith RA and Lam AK: miR-126 in human cancers: Clinical roles and current perspectives. Exp Mol Pathol 96: 98-107, 2014.

17. Meister J and Schmidt MH: miR-126 and miR-126*: New players in cancer. ScientificWorldJournal 10: 2090-2100, 2010.

18. Schober A, Nazari-Jahantigh M, Wei Y, Bidzhekov K, Gremse F, Grommes J, Megens RT, Heyll K, Noels H, Hristov M, et al: MicroRNA-126-5p promotes endothelial proliferation and limits atherosclerosis by suppressing Dlk1. Nat Med 20: 368-376, 2014

19. Esser JS, Saretzki E, Pankratz F, Engert B, Grundmann S, Bode C, Moser M and Zhou Q: Bone morphogenetic protein 4 regulates microRNAs miR-494 and miR-126-5p in control of endothelial cell function in angiogenesis. Thromb Haemost 117: 734-749, 2017

20. Li HY, Zhao X, Liu YZ, Meng Z, Wang D, Yang F and Shi QW: Plasma microRNA-126-5p is associated with the complexity and severity of coronary artery disease in patients with stable angina pectoris. Cell Physiol Biochem 39: 837-846, 2016.
21. Ladeiro Y, Couchy G, Balabaud C, Bioulac-Sage P, Pelletier L, Rebouissou S and Zucman-Rossi J: MicroRNA profiling in hepatocellular tumors is associated with clinical features and oncogene/tumor suppressor gene mutations. Hepatology 47: 1955-1963, 2008

22. Zhang J, Du YY, Lin YF, Chen YT, Yang L, Wang HJ and Ma D: The cell growth suppressor, mir-126, targets IRS-1. Biochem Biophys Res Commun 377: 136-140, 2008.

23. Cho WC, Chow AS and Au JS: Restoration of tumour suppressor hsa-miR-145 inhibits cancer cell growth in lung adenocarcinoma patients with epidermal growth factor receptor mutation. Eur J Cancer 45: 2197-2206, 2009.

24. Yanaihara N, Caplen N, Bowman E, Seike M, Kumamoto K, Yi M, Stephens RM, Okamoto A, Yokota J, Tanaka T, et al: Unique microRNA molecular profiles in lung cancer diagnosis and prognosis. Cancer Cell 9: 189-198, 2006.

25. Felli N, Felicetti F, Lustri AM, Errico MC, Bottero L, Cannistraci A, De Feo A, Petrini M, Pedini F, Biffoni M, et al: miR-126\&126" restored expressions play a tumor suppressor role by directly regulating ADAM9 and MMP7 in melanoma. PLoS One 8: e56824, 2013.

26. Dvorak HF: Vascular permeability factor/vascular endothelial growth factor: A critical cytokine in tumor angiogenesis and a potential target for diagnosis and therapy. J Clin Oncol 20: 4368-4380, 2002.

27. Millauer B, Wizigmann-Voos S, Schnürch H, Martinez R, Møller NP, Risau W and Ullrich A: High affinity VEGF binding and developmental expression suggest Flk-1 as a major regulator of vasculogenesis and angiogenesis. Cell 72: 835-846, 1993.

28. Zeng H, Dvorak HF and Mukhopadhyay D: Vascular permeability factor (VPF)/vascular endothelial growth factor (VEGF) peceptor-1 down-modulates VPF/VEGF receptor-2-mediated endothelial cell proliferation, but not migration, through phosphatidylinositol 3-kinase-dependent pathways. J Biol Chem 276: 26969-26979, 2001

29. Roskoski R Jr: Vascular endothelial growth factor (VEGF) signaling in tumor progression. Crit Rev Oncol Hematol 62: 179-213, 2007.

30. Koch S and Claesson-Welsh L: Signal transduction by vascular endothelial growth factor receptors. Cold Spring Harb Perspect Med 2: a006502, 2012.

31. Chen P, Zhu J, Liu DY, Li HY, Xu N and Hou M: Over-expression of survivin and VEGF in small-cell lung cancer may predict the poorer prognosis. Med Oncol 31: 775, 2014.

32. Ferrara N: The role of vascular endothelial growth factor in pathological angiogenesis. Breast Cancer Res Treat 36: 127-137, 1995.

33. Mattern J, Koomägi R and Volm M: Association of vascular endothelial growth factor expression with intratumoral microvessel density and tumour cell proliferation in human epidermoid lung carcinoma. Br J Cancer 73: 931-934, 1996.

34. Brown LF, Berse B, Jackman RW, Tognazzi K, Manseau EJ, Dvorak HF and Senger DR: Increased expression of vascular permeability factor (vascular endothelial growth factor) and its receptors in kidney and bladder carcinomas. Am J Pathol 143: 1255-1262, 1993.

35. Brown LF, Berse B, Jackman RW, Tognazzi K, Guidi AJ, Dvorak HF, Senger DR, Connolly JL and Schnitt SJ: Expression of vascular permeability factor (vascular endothelial growth factor) and its receptors in breast cancer. Hum Pathol 26: 86-91, 1995.

36. Seto T, Higashiyama M, Funai H, Imamura F, Uematsu K, Seki N, Eguchi K, Yamanaka T and Ichinose Y: Prognostic value of expression of vascular endothelial growth factor and its flt-1 and KDR receptors in stage I non-small-cell lung cancer. Lung Cancer 53: 91-96, 2006.

37. Liu B, Peng XC, Zheng XL, Wang J and Qin YW: miR-126 restoration down-regulate VEGF and inhibit the growth of lung cancer cell lines in vitro and in vivo. Lung Cancer 66: 169-175, 2009.

38. Fearnley GW, Odell AF, Latham AM, Mughal NA, Bruns AF, Burgoyne NJ, Homer-Vanniasinkam S, Zachary IC, Hollstein MC, Wheatcroft SB and Ponnambalam S: VEGF-A isoforms differentially regulate ATF-2-dependent VCAM-1 gene expression and endothelial-leukocyte interactions. Mol Biol Cell 25: 2509-2521, 2014.

39. Shankar S, Ganapathy S, Hingorani SR and Srivastava RK: EGCG inhibits growth, invasion, angiogenesis and metastasis of pancreatic cancer. Front Biosci 13: 440-452, 2008.

40. Kaneshiro T, Morioka T, Inamine M, Kinjo T, Arakaki J, Chiba I, Sunagawa N, Suzui M and Yoshimi N: Anthraquinone derivative emodin inhibits tumor-associated angiogenesis through inhibition of extracellular signal-regulated kinase 1/2 phosphorylation. Eur J Pharmacol 553: 46-53, 2006. 
41. Liu HZ, Yu C, Yang Z, He JL, Chen WJ, Yin J, Li WM, Liu HT and Wang YX: Tubeimoside I sensitizes cisplatin in cisplatin-resistant human ovarian cancer cells (A2780/DDP) through down-regulation of ERK and up-regulation of p38 signaling pathways. Mol Med Rep 4: 985-992, 2011.

42. Dias S, Hattori K, Zhu Z, Heissig B, Choy M, Lane W, Wu Y, Chadburn A, Hyjek E, Gill M, et al: Autocrine stimulation of VEGFR-2 activates human leukemic cell growth and migration. J Clin Invest 106: 511-521, 2000

43. Tian Y, Xie Q, Tian Y, Liu Y, Huang Z, Fan C, Hou B, Sun D, Yao K and Chen T: Radioactive ${ }^{12^{5}} \mathrm{I}$ seed inhibits the cell growth, migration, and invasion of nasopharyngeal carcinoma by triggering DNA damage and inactivating VEGF-A/ERK signaling. PLoS One 8: e74038, 2013.
44. Liu Y, Qiao Y, Hu C, Liu L, Zhou L, Liu B, Chen H and Jiang X: VEGFR2 inhibition by RNA interference affects cell proliferation, migration, invasion, and response to radiation in Calu-1 cells. Clin Transl Oncol 18: 212-219, 2016.

45. Santos SC and Dias S: Internal and external autocrine VEGF/KDR loops regulate survival of subsets of acute leukemia through distinct signaling pathways. Blood 103: 3883-3889, 2004.

This work is licensed under a Creative Commons Attribution-NonCommercial-NoDerivatives 4.0 International (CC BY-NC-ND 4.0) License. 